\title{
Plasma Soluble Lectin-Like Oxidized Low-Density Lipoprotein Receptor-1 as a Novel Prognostic Biomarker in Patients With ST-Segment Elevation Acute Myocardial Infarction
}

Takumi Higuma, MD; Naoki Abe, MD; Syunta Tateyama, MD; Tomohide Endo, MD; Shuji Shibutani, MD; Hiroaki Yokoyama, MD; Kenji Hanada, MD; Masahiro Yamada, MD; Hirofumi Tomita, MD; Hiroyuki Hanada, MD; Tomohiro Osanai, MD; Noriaki Kume, MD; Ken Okumura, MD

Background: Soluble lectin-like oxidized low-density lipoprotein receptor-1 (sLOX-1) level is a reliable prognostic biomarker in acute coronary syndrome. However, it is unclear whether its plasma level at acute phase is related to the long-term prognosis in patients with ST-segment elevation acute myocardial infarction (STEMI).

\begin{abstract}
Methods and Results: We prospectively examined the relation between plasma sLOX-1 level on admission and prognosis in 153 consecutive STEMI patients admitted within $24 \mathrm{~h}$ of onset. Primary percutaneous coronary intervention was performed in 144 patients. The patients were divided into 2 groups by the median value $(71 \mathrm{pg} / \mathrm{ml})$ of plasma sLOX-1 level on admission [sLOX-1 level $\leq 71 \mathrm{pg} / \mathrm{ml}(n=77)$ and $>71 \mathrm{pg} / \mathrm{ml}(\mathrm{n}=76)$ ], and were followed for median of 1,156 days. All-cause mortality and the combined endpoints of major adverse cardiovascular events (MACE) defined as cardiovascular mortality and recurrent MI were both significantly higher in patients with sLOX-1 values above median than in those below median $(25.0 \%$ vs. $3.9 \%, P<0.001$, and $19.4 \%$ vs. $6.5 \%, P=0.019$ by log-rank test, respectively). Even after adjustment for confounders, a level of sLOX-1 above median was an independent predictor for all-cause mortality (hazard ratio (HR): 5.893; 95\% confidence interval (Cl): 1.665-20.854, $\mathrm{P}=0.006$ ) and MACE (HR: 3.457; 95\% Cl: 1.164-10.270, $\mathrm{P}=0.030$ ).
\end{abstract}

Conclusions: Elevated plasma sLOX-1 level on admission independently predicts long-term all-cause mortality and MACE after STEMI. (Circ J 2015; 79: 641-648)

Key Words: Acute coronary syndrome; Biomarkers; Soluble lectin-like oxidized low-density lipoprotein receptor-1 (sLOX-1); ST-segment elevation acute myocardial infarction (STEMI)

$\mathbf{O}$ xidized low-density lipoprotein (Ox-LDL) has been implicated in the pathogenesis of atherosclerosis and atherosclerotic plaque rupture by promoting lipid accumulation, proinflammatory response, and apoptotic cell death, which in turn cause acute coronary syndromes (ACS). ${ }^{1-5}$ Activation of its receptor, lectin-like oxidized low-density lipoprotein receptor 1 (LOX-1), also plays a crucial role in vascular dysfunction, including cell apoptosis and production of matrix metalloproteinase (MMP), which stimulates atherosclerotic plaque rupture and erosion..$^{6-9}$ Indeed, LOX-1 expression is increased by proinflammatory and oxidative stimuli related to atherogenesis, ${ }^{6,10}$ and is prominently enhanced in advanced atherosclerotic plaques of humans. ${ }^{11}$

LOX-1 is not only expressed on the cell surface, but is released as soluble LOX-1 (sLOX-1) into the circulation, where it is proteolytically cleaved at its proximal membrane extracellular domain. ${ }^{12}$ It has been reported that circulating sLOX-1 levels are elevated in patients with ACS, ${ }^{13}$ reflecting its prominent expression and enhanced protease activity in vulnerable atherosclerotic plaques. Moreover, plasma sLOX-1 levels have been shown to be not only a useful biomarker of ACS, ${ }^{13}$ but also a predictor of ACS recurrence or death. ${ }^{14}$ However, it remains unclear how plasma sLOX-1 levels in the acute phase are related to the long-term prognosis in patients with acute myo-

Received August 12, 2014; revised manuscript received December 3, 2014; accepted December 4, 2014; released online January 23, 2015 Time for primary review: 13 days

Department of Cardiology, Hirosaki University Graduate School of Medicine, Hirosaki (T.H., N.A., S.T., T.E., S.S., H.Y., K.H., M.Y., H.T., H.H., T.O., K.O.); Division of Clinical Pharmacy, Faculty of Pharmaceutical Sciences, Kobe Gakuin University, Kobe (N.K.), Japan

Mailing address: Ken Okumura, MD, Department of Cardiology, Hirosaki University Graduate School of Medicine, 5 Zaifu-cho, Hirosaki 036-8562, Japan. E-mail: okumura@cc.hirosaki-u.ac.jp

ISSN-1346-9843 doi:10.1253/circj.CJ-14-0904

All rights are reserved to the Japanese Circulation Society. For permissions, please e-mail: cj@j-circ.or.jp 
cardial infarction (AMI). In the present study, we conducted a prospective clinical study examining the relationship between plasma sLOX-1 level on admission and prognosis in patients with ST-segment elevation AMI (STEMI).

\section{Methods}

\section{Study Patients}

The study protocol was approved by the institutional Ethics Committee on Human Research, and the informed consent was given by all patients. This was a prospective observational study of patients consecutively admitted from February 2008 to April 2009 within $24 \mathrm{~h}$ of symptom onset with a diagnosis of STEMI who underwent emergency coronary angiography. STEMI was defined as an elevated creatine kinase myocardial isoform (CK-MB) and/or cardiac troponin T value greater than upper reference limit, with 2 of the following: (1) typical chest pain lasting $>20$ min and (2) ECG showing new ST-segment elevation $\geq 2 \mathrm{~mm}$ in at least 2 contiguous precordial leads or $\geq 1 \mathrm{~mm}$ in at least 2 contiguous limb leads, or newly apparent left bundle branch block. All patients consented to emergency coronary angiography to make the definite diagnosis and assess the severity of coronary artery disease in the acute phase.

\section{Measurements of sLOX-1 and Other Biomarkers}

On admission and before emergency coronary angiography, peripheral blood samples were collected in tubes containing ethylenediaminetetraacetic acid dipotassium salt to separate the plasma. The samples were centrifuged for $10 \mathrm{~min}$ and stored at $-80^{\circ} \mathrm{C}$ until analyzed. Plasma was isolated from the blood immediately after sample withdrawal to avoid the possibility of changes in SLOX-1 during the time period of plasma isolation. Circulating sLOX-1 levels in the plasma were measured as previously described. ${ }^{15}$ Briefly, sLOX-1 levels were measured by a sandwich chemiluminescent enzyme immunoassay (Shionogi Co, Ltd, Osaka, Japan) using 2 different human LOX-1-specific monoclonal antibodies, one of which was labeled with a chemiluminescent agent, and recombinant human LOX-1 extracellular domain as an assay standard. Intra- and interassay coefficients of variation were reported to be 4.4$6.5 \%$ and $4.0-6.1 \%$, respectively, and the lower limit of detection for sLOX-1 was $8.0 \mathrm{pg} / \mathrm{ml}^{15}$

Lipids, glucose, HbA1c, and other biomarkers were measured with standard hospital assays immediately after sampling. Left ventricular ejection fraction (LVEF) was assessed by contrast left ventriculography or 2-dimensional transthoracic echocardiography performed immediately after emergency percutane-

\begin{tabular}{|c|c|c|c|c|}
\hline & All $(n=153)$ & $\begin{array}{c}\text { sLOX-1 } \leq 71 \mathrm{pg} / \mathrm{ml} \\
(\mathrm{n}=77)\end{array}$ & $\begin{array}{c}\text { sLOX-1 > } 71 \mathrm{pg} / \mathrm{ml} \\
(\mathrm{n}=76)\end{array}$ & $P$ value \\
\hline \multicolumn{5}{|l|}{ Clinical characteristics } \\
\hline Age, years & $67 \pm 12$ & $68 \pm 13$ & $66 \pm 12$ & 0.469 \\
\hline Male sex, n (\%) & $115(75)$ & $60(78)$ & $55(72)$ & 0.459 \\
\hline $\mathrm{BMI}, \mathrm{kg} / \mathrm{m}^{2}$ & $24.4 \pm 3.4$ & $24.5 \pm 3.7$ & $24.3 \pm 3.1$ & 0.692 \\
\hline \multicolumn{5}{|l|}{ Coronary risk factor } \\
\hline Hypertension, n (\%) & $115(75)$ & $57(74)$ & $58(76)$ & 0.852 \\
\hline Diabetes mellitus, n (\%) & $51(33)$ & $24(31)$ & $27(36)$ & 0.609 \\
\hline Dyslipidemia, n (\%) & $96(63)$ & $49(64)$ & $47(62)$ & 0.868 \\
\hline Current smoking, n (\%) & $83(54)$ & $44(57)$ & $39(51)$ & 0.518 \\
\hline Previous MI, n (\%) & $7(5)$ & $5(7)$ & $2(3)$ & 0.442 \\
\hline Previous CABG, n (\%) & $4(3)$ & $3(4)$ & $1(1)$ & 0.620 \\
\hline Previous PCI, n (\%) & $8(5)$ & $6(8)$ & $2(3)$ & 0.276 \\
\hline Time from onset to $E R$, min & $240(152-387)$ & $240(172-360)$ & $240(136-401)$ & 0.558 \\
\hline Killip class $\geq 2, n(\%)$ & $30(20)$ & $13(17)$ & $17(22)$ & 0.422 \\
\hline LVEF, \% & $46 \pm 11$ & $47 \pm 10$ & $45 \pm 12$ & 0.540 \\
\hline \multicolumn{5}{|l|}{ Angiographic characteristics } \\
\hline \multicolumn{5}{|l|}{ No. of diseased vessels } \\
\hline SVD, n (\%) & $58(38)$ & $26(34)$ & $32(42)$ & 0.508 \\
\hline DVD, n (\%) & $50(33)$ & $28(36)$ & $22(29)$ & \\
\hline TVD, n (\%) & $45(29)$ & $23(30)$ & $22(29)$ & \\
\hline \multicolumn{5}{|l|}{ Infarct-related artery } \\
\hline $\mathrm{RCA}, \mathrm{n}(\%)$ & $54(35)$ & $26(34)$ & $28(37)$ & 0.492 \\
\hline LMT, n (\%) & $1(1)$ & $1(1)$ & $0(0)$ & \\
\hline LAD, n (\%) & $80(52)$ & $43(56)$ & $37(49)$ & \\
\hline LCX, n (\%) & $18(12)$ & $7(9)$ & $11(45)$ & \\
\hline \multicolumn{5}{|l|}{ Initial TIMI flow } \\
\hline $0, \mathrm{n}(\%)$ & $106(69)$ & $51(66)$ & $55(72)$ & 0.378 \\
\hline $1, \mathrm{n}(\%)$ & $14(9)$ & $10(13)$ & $4(5)$ & \\
\hline $2, \mathrm{n}(\%)$ & $23(15)$ & $12(16)$ & $11(15)$ & \\
\hline $3, \mathrm{n}(\%)$ & $10(7)$ & $4(5)$ & $6(8)$ & \\
\hline Emergency PCl, n (\%) & $144(94)$ & 76 (99) & $68(90)$ & 0.018 \\
\hline
\end{tabular}




\begin{tabular}{|c|c|c|c|c|}
\hline \multicolumn{5}{|l|}{ Biochemical data } \\
\hline eGFR, $\mathrm{ml} \cdot \mathrm{min}^{-1} \cdot 1.73 \mathrm{~m}^{-2}$ & $70 \pm 23$ & $75 \pm 22$ & $64 \pm 22$ & 0.004 \\
\hline \multicolumn{5}{|l|}{ Lipids } \\
\hline Total cholesterol, mg/dl & $197 \pm 43$ & $198 \pm 43$ & $196 \pm 43$ & 0.675 \\
\hline Triglycerides, mg/dl & $125 \pm 74$ & $117 \pm 60$ & $131 \pm 84$ & 0.244 \\
\hline HDL-C, mg/dl & $47 \pm 13$ & $47 \pm 13$ & $48 \pm 13$ & 0.697 \\
\hline LDL-C, mg/dl & $125 \pm 39$ & $128 \pm 40$ & $123 \pm 38$ & 0.403 \\
\hline Glucose, mg/dl & $153(121-205)$ & $131(115-188)$ & $179(133-244)$ & $<0.001$ \\
\hline $\mathrm{HbA} 1 \mathrm{c}, \%$ & $6.1 \pm 1.1$ & $6.0 \pm 1.1$ & $6.2 \pm 1.2$ & 0.401 \\
\hline $\mathrm{BNP}, \mathrm{pg} / \mathrm{dl}$ & $58.5(25-172)$ & $58.0(25.5-124.5)$ & $65.5(25.3-219.0)$ & 0.588 \\
\hline hs-CRP, $\mu \mathrm{g} / \mathrm{dl}$ & 169 (573-599) & $153(52-658)$ & $195(65-587)$ & 0.339 \\
\hline CPK, IU/L & $266(141-777)$ & $276(134-847)$ & $263(155-725)$ & 0.881 \\
\hline CK-MB, IU/L & $25(13-70)$ & $28(14-72)$ & $25(12-74)$ & 0.865 \\
\hline Troponin $\mathrm{T}, \mathrm{ng} / \mathrm{ml}(\mathrm{n}=152)$ & $0.19(0.05-0.65)$ & $0.18(0.06-0.56)$ & $0.25(0.04-0.88)$ & 0.669 \\
\hline Troponin $\mathrm{T}>0.1 \mathrm{ng} / \mathrm{ml}, \mathrm{n}(\%)(\mathrm{n}=152)$ & $99(65)$ & $51(66)$ & $48(62)$ & 0.410 \\
\hline Peak CPK, IU/L ( $n=149)$ & $2,443(1,131-4,451)$ & $1,928(775-3,944)$ & $3,128(1,367-4,980)$ & 0.016 \\
\hline Peak CK-MB, IU/L ( $n=149)$ & $213(93-378)$ & $165(71-375)$ & $264(101-407)$ & 0.087 \\
\hline \multicolumn{5}{|l|}{ Medications on admission } \\
\hline Aspirin, $\mathrm{n}(\%)$ & $22(14)$ & $11(14)$ & $11(15)$ & 1.000 \\
\hline Clopidogrel, n (\%) & $2(1)$ & $1(1)$ & $1(1)$ & 1.000 \\
\hline ACEI or ARB, $n(\%)$ & $44(29)$ & $21(27)$ & $23(30)$ & 0.723 \\
\hline$\beta$-blocker, $\mathrm{n}(\%)$ & $14(9)$ & $8(10)$ & $6(8)$ & 0.780 \\
\hline Statin, n (\%) & $23(15)$ & $12(16)$ & $11(15)$ & 1.000 \\
\hline Aldosterone receptor antagonist, $\mathrm{n}(\%)$ & $3(2)$ & $1(1)$ & $2(3)$ & 0.618 \\
\hline Pioglitazone, n (\%) & $5(3)$ & $3(4)$ & $2(3)$ & 0.618 \\
\hline Medications at discharge $(n=145)$ & & $(n=76)$ & $(n=69)$ & \\
\hline Aspirin, $\mathrm{n}(\%)$ & $145(100)$ & $76(100)$ & $69(100)$ & 1.000 \\
\hline Clopidogrel, n (\%) & $138(95)$ & $74(97)$ & $64(93)$ & 0.258 \\
\hline ACEI or ARB, $n(\%)$ & $130(90)$ & $71(93)$ & $59(86)$ & 0.171 \\
\hline$\beta$-blocker, $\mathrm{n}(\%)$ & $126(87)$ & $63(83)$ & $63(91)$ & 0.148 \\
\hline Statin, n (\%) & $131(90)$ & $69(91)$ & $62(90)$ & 1.000 \\
\hline Aldosterone receptor antagonist, $\mathrm{n}(\%)$ & $12(8)$ & $4(5)$ & $8(12)$ & 0.230 \\
\hline Pioglitazone, n (\%) & $13(9)$ & $8(11)$ & $5(7)$ & 0.569 \\
\hline
\end{tabular}

Data are presented as frequency (percentage) for categorical variables and as mean \pm standard deviation or median (interquartile range) for continuous variables.

ACEI, angiotensin converting enzyme inhibitor; ARB, angiotensin II receptor blocker; BMI, body mass index; BNP, B-type natriuretic peptide; CABG, coronary artery bypass grafting; CK-MB, creatine kinase myocardial isoform; CPK, creatine phosphokinase; DVD, double-vessel disease; eGFR, estimated glomerular filtration rate; ER, emergency room; HDL-C, high-density lipoprotein cholesterol; hs-CRP, high-sensitivity C-reactive protein; LAD, left anterior descending artery; LCX, left circumflex artery; LDL-C, low-density lipoprotein cholesterol; LMT, left main coronary artery; LVEF, left ventricular ejection fraction; MI, myocardial infarction; PCI, percutaneous coronary intervention; RCA, right coronary artery; sLOX-1, soluble lectin-like-oxidized low-density lipoprotein receptor-1; SVD, single vessel disease; TIMI, Thrombolysis in Myocardial Infarction; TVD, triple-vessel disease.

ous coronary intervention (PCI) or coronary angiography.

\section{Follow-up}

The endpoints of this study were all-cause mortality and the combined endpoint of major adverse cardiovascular events (MACE), which was defined as cardiovascular mortality and recurrent nonfatal MI. Follow-up started on the day of admission and the patients were prospectively followed for 3 years (median, 1,156 days) with optimal medical treatment. Followup data were obtained as follows: reviewing patients' hospital records, interviewing the patients by telephone, and examining the patients at outpatient clinics.

\section{Statistical Analysis}

The patients were divided into 2 groups based on the median value of plasma SLOX-1 on admission: those with SLOX-1 level below and above the median. Because this study was exploratory, no power analysis or sample size estimate was performed in advance. Kaplan-Meier curves for all-cause mortality and MACE were compared between patients with plasma sLOX-1 levels below and above the median and were evaluated by the log-rank test. Comparisons between groups were performed by Pearson's chi-square test or Fisher exact for categorical variables when appropriate. Continuous variables were compared using Student's t test, if normally distributed, and MannWhitney U test, if non-normally distributed. All data are expressed as mean \pm standard deviation or median and interquartile range. Correlations between sLOX-1 and other continuous variables were examined by a nonparametric (Spearman) correlation coefficient. Univariate analyses were performed using Cox proportional hazard model to identify the factors that significantly predicted all-cause mortality and MACE. The difference in risk was expressed as the hazard ratio (HR) with corresponding $95 \%$ confidence interval (CI). The following baseline variables were included in the univariate analysis: age, sex, Killip classification $\geq 2$, emergency PCI, LVEF, hypertension, diabe- 


\begin{tabular}{|c|c|c|}
\hline \multicolumn{3}{|c|}{$\begin{array}{l}\text { Table 2. Spearman Correlation Coefficients }(R) \text { Between } \\
\text { Plasma sLOX-1 Levels and Continuous Variables } \\
(n=153) \text { in the Study Group of Patients With STEMI }\end{array}$} \\
\hline & Spearman $\mathbf{R}$ & $P$ value \\
\hline Age, years & -0.109 & 0.179 \\
\hline $\mathrm{BMI}, \mathrm{kg} / \mathrm{m}^{2}$ & 0.017 & 0.837 \\
\hline Time from onset to $E R$, $\min$ & -0.096 & 0.239 \\
\hline LVEF, \% & -0.082 & 0.311 \\
\hline eGFR, $\mathrm{ml} \cdot \mathrm{min}^{-1} \cdot 1.73 \mathrm{~m}^{-2}$ & -0.159 & 0.049 \\
\hline Triglycerides, mg/dl & 0.096 & 0.237 \\
\hline LDL-C, mg/dl & -0.025 & 0.755 \\
\hline $\mathrm{HDL}-\mathrm{C}, \mathrm{mg} / \mathrm{dl}$ & -0.058 & 0.480 \\
\hline Glucose, mg/dl & 0.349 & $<0.001$ \\
\hline $\mathrm{HbA1c}, \%$ & 0.075 & 0.357 \\
\hline $\mathrm{BNP}, \mathrm{pg} / \mathrm{ml}$ & 0.029 & 0.725 \\
\hline hs-CRP, mg/dl & 0.147 & 0.070 \\
\hline CPK, IU/L & 0.031 & 0.699 \\
\hline CK-MB, IU/L & -0.004 & 0.959 \\
\hline Troponin $\mathrm{T}, \mathrm{ng} / \mathrm{ml}(\mathrm{n}=152)$ & 0.036 & 0.660 \\
\hline Peak CPK, IU/L (n=149) & 0.210 & 0.010 \\
\hline Peak CK-MB, IU/L $(n=149)$ & 0.151 & 0.064 \\
\hline
\end{tabular}

STEMI, ST-segment elevation acute myocardial infarction. Other abbreviations as in Table 1.

tes mellitus, lipid parameters including low-density lipoprotein cholesterol, high-density lipoprotein cholesterol, and triglycerides, estimated glomerular filtration rate (eGFR), glucose, peak creatine phosphokinase (CPK), high-sensitivity C-reactive protein (hs-CRP), and plasma sLOX-1. Because serum glucose, peak CPK, hs-CRP, and plasma sLOX-1 levels were not normally distributed, they were logarithmically transformed. Plasma sLOX-1 was also analyzed both as a continuous and a categorical variable based on the median value. We performed multivariate Cox proportional hazard analysis to elucidate in- dependent predictors for the incidences of all-cause mortality and MACE. Only variables that had a P-value $<0.10$ in univariate analysis were considered in the multivariate Cox proportional hazard model using backward elimination techniques. Receiver-operating characteristic (ROC) analysis was performed to determine optimal cut-off values of plasma sLOX-1 to predict all-cause mortality and MACE. For the statistical analysis, $\mathrm{P}$ values $<0.05$ were considered of statistical significance. Data were statistically analyzed using the SPSS software package version 20.0 (SPSS, Chicago, IL, USA).

\section{Results}

\section{Patients' Profiles}

The study group comprised 153 consecutive patients ( 115 males, 38 females; mean age, $67 \pm 12$ years). The median ( 25 th to 75 th percentiles) plasma level of sLOX-1 was $71(59.0-95.5) \mathrm{pg} / \mathrm{ml}$. All patients had plasma sLOX-1 levels above the lower limit of detection. The patients were divided into 2 groups based on this value $(71 \mathrm{pg} / \mathrm{ml})$ of plasma sLOX-1 at admission: those with sLOX-1 level $\leq 71 \mathrm{pg} / \mathrm{ml}(\mathrm{n}=77)$ and $>71 \mathrm{pg} / \mathrm{ml}(\mathrm{n}=76)$. The baseline characteristics of the patients in the 2 groups are summarized in Table 1. Of these patients, 9 did not undergo emergency PCI on the decision of the operator because of free-wall cardiac rupture before PCI $(n=4)$, small vessel native coronary occlusion $(n=3)$, and spontaneous reperfusion before PCI $(n=2)$, and therefore 144 had an emergency PCI in the acute phase. Emergency PCI was performed more frequently in patients with SLOX-1 levels below the median than in those with a level above the median ( $99 \%$ vs. $90 \%, \mathrm{P}=0.018)$. $\mathrm{Pa}-$ tients with a sLOX-1 level above the median had significantly reduced eGFR $(\mathrm{P}=0.004)$ and higher glucose levels $(\mathrm{P}<0.001)$ compared with those below the median level on admission. The peak CPK levels were significantly higher in patients with sLOX-1 levels above the median than in those below the median $(\mathrm{P}=0.016)$. There were no significant differences in the other characteristics of the 2 groups, including coronary risk factors, distribution of infarct-related artery, the number of sig-
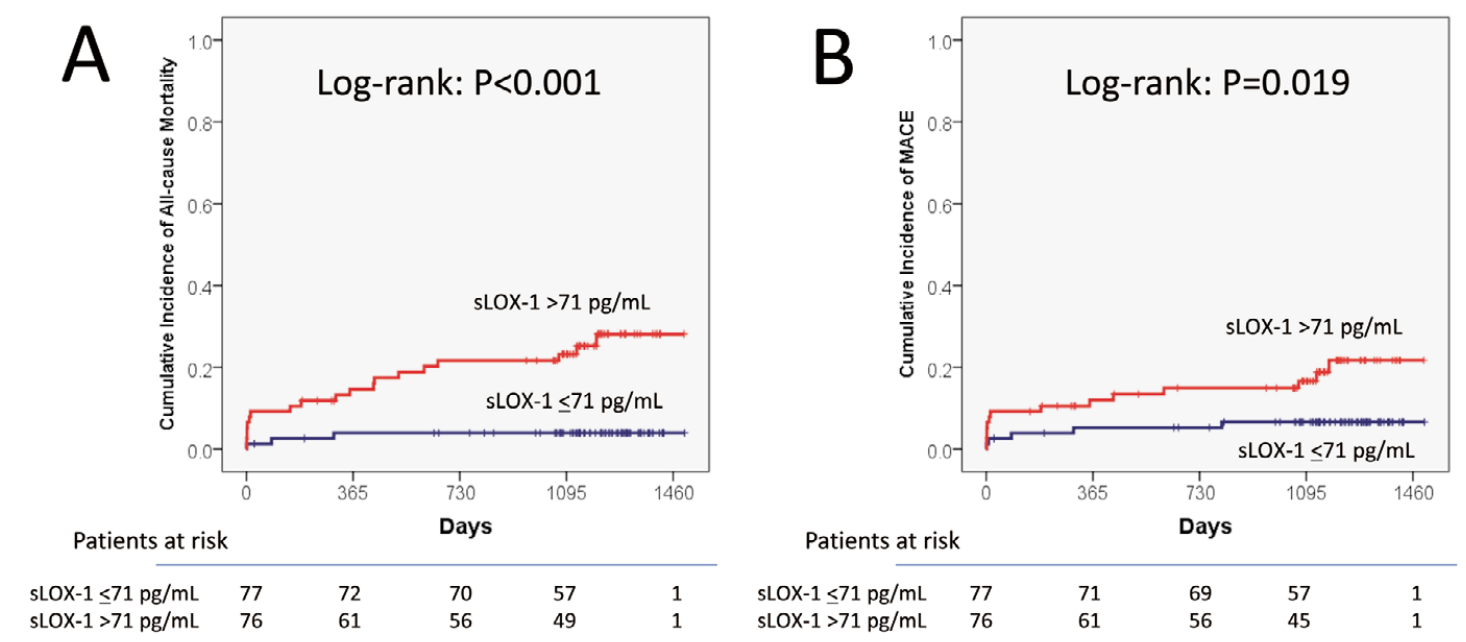

Figure. Comparison of Kaplan-Meier curves for all-cause mortality (A) and major adverse cardiac events (B) between patients with sLOX-1 values above and below the median. Cumulative incidence curves of STEMI patients with plasma sLOX-1 values above median $(71 \mathrm{pg} / \mathrm{ml}$ ) (red line) and below (blue line) are shown. sLOX-1, soluble lectin-like oxidized low-density lipoprotein receptor-1; STEMI, ST-segment elevation acute myocardial infarction. 
Table 3. Predictors of All-Cause Mortality and MACE (Univariate Analysis) in the Study Group of Patients With STEMI

\begin{tabular}{|c|c|c|c|c|c|c|}
\hline \multirow{2}{*}{ Factor } & \multicolumn{3}{|c|}{ All-cause mortality $(n=22)$} & \multicolumn{3}{|c|}{ MACE $(n=19)$} \\
\hline & HR & $95 \% \mathrm{Cl}$ & $P$ value & HR & $95 \% \mathrm{Cl}$ & $P$ value \\
\hline Age (per 1 year increase) & 1.074 & $1.028-1.123$ & 0.001 & 1.086 & $1.033-1.141$ & 0.001 \\
\hline Male sex & 0.449 & $0.192-1.052$ & 0.065 & 0.428 & $0.172-1.066$ & 0.068 \\
\hline Killip class $\geq 2$ & 3.844 & $1.659-8.905$ & 0.002 & 2.690 & $1.058-6.843$ & 0.038 \\
\hline Emergency $\mathrm{PCl}$ & 0.200 & $0.067-0.593$ & 0.004 & 0.242 & $0.070-0.834$ & 0.025 \\
\hline LVEF (per $1 \%$ increase) & 0.932 & $0.898-0.967$ & $<0.001$ & 0.95 & $0.914-0.991$ & 0.015 \\
\hline Hypertension & 0.841 & $0.329-2.150$ & 0.718 & 0.675 & $0.257-1.777$ & 0.426 \\
\hline Diabetes mellitus & 1.145 & $0.480-2.730$ & 0.759 & 0.924 & $0.351-2.431$ & 0.873 \\
\hline LDL-C (per 1 mg/dl increase) & 0.994 & $0.982-1.006$ & 0.324 & 0.997 & $0.985-1.010$ & 0.690 \\
\hline HDL-C (per $1 \mathrm{mg} / \mathrm{dl}$ increase) & 1.006 & $0.976-1.037$ & 0.696 & 1.004 & $0.972-1.038$ & 0.798 \\
\hline Triglycerides (per $1 \mathrm{mg} / \mathrm{dl}$ increase) & 0.993 & $0.985-1.001$ & 0.105 & 0.994 & $0.986-1.002$ & 0.166 \\
\hline eGFR (per $\left.1 \mathrm{ml} \cdot \mathrm{min}^{-1} \cdot 1.73 \mathrm{~m}^{-2}\right)$ & 0.953 & $0.934-0.973$ & $<0.001$ & 0.961 & $0.941-0.982$ & $<0.001$ \\
\hline Log glucose & 7.074 & $2.322-21.556$ & 0.011 & 4.667 & $1.431-15.221$ & 0.011 \\
\hline Log peak CPK & 1.294 & $0.784-2.137$ & 0.313 & 0.987 & $0.597-1.633$ & 0.961 \\
\hline Log hs-CRP & 1.625 & $1.265-2.086$ & $<0.001$ & 1.310 & $0.996-1.724$ & 0.054 \\
\hline sLOX-1 (>71 pg/ml) & 7.279 & $2.152-24.623$ & 0.001 & 3.171 & $1.140-8.817$ & 0.027 \\
\hline Log sLOX-1 & 5.014 & $2.588-9.715$ & $<0.001$ & 2.733 & $1.171-6.382$ & 0.020 \\
\hline
\end{tabular}

$\mathrm{Cl}$, confidence interval; HR, hazard ratio; MACE, major adverse cardiovascular events. Other abbreviations as in Tables 1,2.

\begin{tabular}{|c|c|c|c|}
\hline Factor & HR & $95 \% \mathrm{Cl}$ & $P$ value \\
\hline \multicolumn{4}{|l|}{ All-cause mortality } \\
\hline Age (per 1 year increase) & 1.089 & $1.029-1.152$ & 0.003 \\
\hline eGFR (per $1 \mathrm{ml} \cdot \mathrm{min}^{-1} \cdot 1.73 \mathrm{~m}^{-2}$ increase) & 0.979 & $0.960-0.999$ & 0.036 \\
\hline Log hs-CRP & 1.386 & $1.044-1.841$ & 0.024 \\
\hline sLOX-1 (>71 pg/ml) & 5.893 & $1.665-20.854$ & 0.006 \\
\hline \multicolumn{4}{|l|}{ MACE } \\
\hline Age (per 1 year increase) & 1.090 & $1.029-1.154$ & 0.003 \\
\hline eGFR (per $1 \mathrm{ml} \cdot \mathrm{min}^{-1} \cdot 1.73 \mathrm{~m}^{-2}$ increase) & 0.976 & $0.954-0.998$ & 0.035 \\
\hline sLOX-1 (>71 pg/ml) & 3.457 & $1.164-10.270$ & 0.026 \\
\hline
\end{tabular}

Abbreviations as in Tables 1-3.

nificantly stenotic coronary arteries, Killip classification, LVEF, hs-CRP, troponin T, B-type natriuretic peptide (BNP), and peak CK-MB.

Correlations of plasma sLOX-1 level with various parameters were studied (Table 2). Biomarkers such as troponin $\mathrm{T}$, $\mathrm{BNP}$, and hs-CRP were not significantly correlated with plasma sLOX-1. Only eGFR ( $\mathrm{r}=-0.159, \mathrm{P}=0.049)$, glucose $(\mathrm{r}=0.349$, $\mathrm{P}<0.001)$, and peak $\mathrm{CPK}(\mathrm{r}=0.210, \mathrm{P}=0.010)$ showed relatively weak, but statistically significant correlations.

\section{Prognostic Value of Plasma sLOX-1 Level}

During the follow-up period (median: 1,156 days), all-cause mortality was $14.3 \%(n=22)$, including $9.8 \%(n=16)$ cardiovascular causes; $3.3 \%(n=5)$ of the patients had recurrent MI, and $12.4 \%(\mathrm{n}=19)$ experienced MACE. Kaplan-Meier analyses showed that all-cause mortality (Figure A) and MACE (Figure B) were both significantly higher in patients with sLOX-1 values above the median than in those with levels below the median $(25.0 \%$ vs. $3.9 \%, \mathrm{P}<0.001$ and $19.4 \%$ vs. $6.5 \%, \mathrm{P}=0.019$ by log-rank test, respectively).

To evaluate the role of plasma sLOX-1 as a short-term and long-term prognostic factor, 30-day Kaplan-Meier analyses and after 30 days were performed separately. All-cause mortality in 30 days and after 30 days were both significantly higher in patients with sLOX-1 values above the median than in those below the median $(9.2 \%$ vs. $1.3 \%, \mathrm{P}=0.029$ and $20.8 \%$ vs. $2.7 \%, \mathrm{P}=0.002$ by log-rank test, respectively). There were no significant differences in MACE either in 30 days or after 30 days between patients with sLOX-1 above and below the median $(9.2 \%$ vs. $2.6 \%, \mathrm{P}=0.084$ and $13.8 \%$ vs. $4.2 \%, \mathrm{P}=0.113$ by log-rank test, respectively).

\section{Univariate and Multivariate Analyses of All-Cause Mortality and MACE}

Univariate analysis showed age, Killip classification $\geq 2$, emergency PCI, LVEF, eGFR, and log glucose were significantly associated with both all-cause mortality and MACE (Table 3). Plasma sLOX-1 level, when analyzed either as a continuous or a categorical variable, was also associated with both allcause mortality and MACE. Log hs-CRP was significantly associated with all-cause mortality. The other variables, including hypertension, diabetes mellitus, or lipid parameters, showed no significant relationships with the incidence of all-cause mortality or MACE.

Multivariate Cox proportional hazard analysis showed that patients with plasma sLOX-1 levels above the median 


\begin{tabular}{|c|c|c|c|}
\hline Factor & HR & $95 \% \mathrm{Cl}$ & $P$ value \\
\hline \multicolumn{4}{|l|}{ All-cause mortality } \\
\hline Age (per 1 year increase) & 1.083 & $1.025-1.144$ & 0.005 \\
\hline eGFR (per $1 \mathrm{ml} \cdot \mathrm{min}^{-1} \cdot 1.73 \mathrm{~m}^{-2}$ increase) & 0.979 & $0.958-0.999$ & 0.042 \\
\hline Log hs-CRP & 1.536 & $1.159-2.036$ & 0.003 \\
\hline Log sLOX-1 & 3.743 & $1.853-7.562$ & $<0.001$ \\
\hline \multicolumn{4}{|l|}{ MACE } \\
\hline Age (per 1 year increase) & 1.083 & $1.024-1.145$ & 0.005 \\
\hline eGFR (per $1 \mathrm{ml} \cdot \mathrm{min}^{-1} \cdot 1.73 \mathrm{~m}^{-2}$ increase) & 0.974 & $0.952-0.997$ & 0.027 \\
\hline Log sLOX-1 & 2.436 & $1.066-5.566$ & 0.035 \\
\hline
\end{tabular}

Abbreviations as in Tables 1-3.

$(>71 \mathrm{pg} / \mathrm{ml})$ were significantly associated with higher incidences of all-cause mortality (HR: 5.893; 95\% CI: 1.665-20.854, $\mathrm{P}=0.006)$ and MACE (HR: 3.457; 95\% CI: 1.164-10.270, $\mathrm{P}=0.030$ ) (Table 4). As a continuous variable, log plasma sLOX-1 level was also associated with all-cause mortality (HR: 3.743; 95\% CI: 1.853-7.562, P<0.001) and MACE (HR: 2.436; 95\% CI: 1.066-5.566, $\mathrm{P}=0.035$ ) (Table 5).

\section{ROC Analysis of Plasma sLOX-1 for Predicting All-Cause Mortality and MACE}

The plasma SLOX-1 level to predict all-cause mortality had an area under the curve (AUC) of 0.751, and its best cut-off value was $74 \mathrm{pg} / \mathrm{ml}$, with a sensitivity of $86.4 \%$ and a specificity of $59.5 \%$. Furthermore, plasma sLOX-1 level to predict MACE had an AUC of 0.629, and its best cut-off value was $78.5 \mathrm{pg} / \mathrm{ml}$, with a sensitivity of $68.4 \%$ and a specificity of $63.4 \%$.

\section{Discussion}

\section{Major Findings}

The present study showed that a higher level of plasma sLOX-1 measured on admission in patients with STEMI is an unfavorable prognostic factor for long-term all-cause mortality and MACE. Furthermore, multivariate analysis demonstrated that both a plasma sLOX-1 level above the median and each 1 log plasma sLOX-1 increase are independently associated with all-cause mortality and MACE after adjusting for confounders in STEMI. Despite numerous technical advances regarding early diagnosis and treatment such as PCI, patients with STEMI remain at a high risk for death, ${ }^{16-19}$ particularly when accompanied by cardiogenic shock, severe ventricular arrhythmia, or cardiac rupture. The present findings seem to be useful for identifying those patients requiring more aggressive therapy and intensive clinical management.

\section{Plasma sLOX-1 Level as a Novel Prognostic Marker in Patients With STEMI}

LOX-1 was initially identified as a cell-surface receptor for Ox-LDL in endothelial cells, but was also shown to be expressed on macrophages and smooth muscle cells. For example, LOX-1 is prominently expressed on the surface of intimal smooth muscle cells and lipid-laden macrophages in human atherosclerotic lesions. ${ }^{11}$ Furthermore, LOX-1 plays important roles in Ox-LDL-induced apoptosis of vascular endothelial and smooth muscle cells, ${ }^{6-8}$ and production of MMP, ${ }^{9}$ which evokes plaque vulnerability and rupture, and therefore its expression is closely associated with instability of atherosclerotic plaques. LOX-1 is cleaved from the cell surface by certain proteases, and released as sLOX $-1 .{ }^{12}$ A previous clinical study demonstrated that circulating SLOX-1 levels are specifically elevated in ACS, reflecting its prominent expression and enhanced protease activities in vulnerable plaques, and that sLOX-1 can be a useful diagnostic biomarker of early ACS. ${ }^{13}$ A recent study with optical coherence tomography during the acute phase of ACS further showed that elevated levels of plasma SLOX-1 were significantly associated with plaque rupture and thin-cap fibroatheroma. ${ }^{20}$ These previous studies indicated the usefulness of sLOX-1 as a diagnostic marker for plaque instability, not for cardiac necrosis or injury. Indeed, the present study found no correlations between plasma SLOX-1 level and troponin $\mathrm{T}$ or CK-MB level on admission, both of which are established markers for cardiac necrosis or injury.

A previous study ${ }^{14}$ already suggested the potential value of sLOX-1 in the prognostic stratification of patients with ACS. However, a smaller heterogeneous population of patients with ACS including STEMI and non-ST-segment elevation (NSTE)ACS was studied ( $n=94)$, with a shorter follow-up period (median, 896 days). NSTE-ACS is pathophysiologically different from STEMI. STEMI results from complete and prolonged occlusion of the coronary artery, whereas NSTE-ACS usually from severe narrowing of the coronary artery or its transient occlusion by thrombus and/or atheromatous plaques. To the best of our knowledge, the present study is the largest prospective observational study in patients with STEMI, a typical and homogeneous entity of ACS, to evaluate the long-term prognostic value of plasma SLOX-1. sLOX-1 levels were quite different in the study by Kume et al ${ }^{14}$ and our present study. These differences may be related to different samples or assay performance. Kume et al measured serum sLOX-1 using a previously described enzyme-linked immunosorbent assay with standard polyclonal antibodies, ${ }^{13,14}$ whereas we measured plasma sLOX-1 levels using a sandwich chemiluminescent enzyme immunoassay with 2 newly developed anti-human LOX-1 monoclonal antibodies. ${ }^{15}$

In the present study, log hs-CRP was also identified as a significant and independent marker for all-cause mortality. A major limitation of hs-CRP is its lack of specificity. Elevated hs-CRP levels as a systemic acute phase reactant are often observed under conditions such as infection other than atherosclerosis. sLOX-1 is elevated in the acute phase of ACS, but not in general acute inflammatory diseases in which hs-CRP levels are high. ${ }^{13}$ Therefore, sLOX-1 may be a useful prognostic biomarker even in ACS patients complicated by acute inflammatory diseases. 
The infarct size assessed by peak CPK or peak CK-MB is a powerful predictor of poor prognosis after STEMI. In the present study, however, the log peak CPK showed no significant relationship with the incidence of all-cause mortality or MACE. Peak CPK values were missing for the 3 patients who died during the acute phase of STEMI and this might have influenced the results of the statistical analysis.

\section{Possible Mechanism of Elevated Plasma sLOX-1 Levels in Patients With STEMI}

The mechanisms of the induction and regulation of circulating sLOX-1 levels in STEMI could not be fully evaluated in the present study. We found no association between patients with a plasma SLOX-1 above the median and the severity of AMI as determined by the distribution of the infarct-related artery, the number of significantly stenotic coronary arteries, Killip classification, LVEF, or CPK at admission, even though high sLOX-1 independently predicted all-cause mortality and MACE. On the other hand, peak CPK level was significantly higher in patients with sLOX-1 levels above the median than in those below the median. Kobayashi et al also reported that peak CK-MB values were higher in ACS patients with SLOX-1 above their cut-off value than in those below the cut-off value. ${ }^{21}$ This may be explained by delayed myocardial damage from inflammatory processes or reperfusion injury mediated by SLOX-1. The levels of mRNA LOX-1 and LOX-1 protein were markedly upregulated in the left ventricle of salt-sensitive Dahl rats with hypertensive heart failure. ${ }^{22}$ Moreover, a previous study demonstrated that the expression of LOX-1 can be induced in cardiac myocytes and that activation of this pathway induced apoptosis via a p38 mitogen-activated protein kinase pathway. ${ }^{23}$ In a rat model of ischemia-reperfusion, the administration of anti-LOX-1 antibody prevented cardiac remodeling by reducing the infarct size and improving left ventricular function through inhibition of apoptosis and oxidative stress in cardiac myocytes. ${ }^{24}$ These studies suggest that LOX-1 plays an important role in myocardial ischemia, in terms of determination of left ventricular function and infarct size. Elevated sLOX-1 in the acute phase of STEMI has, therefore, been proposed as not only an early diagnostic marker but also a marker of disease progression in patients with STEMI. Indeed, we found a positive correlation between levels of plasma SLOX-1 and admission glucose, probably because high glucose levels induce the expression of LOX-1, as previously shown by an in vitro study. ${ }^{25}$

\section{Relationship Between Plasma sLOX-1 Level and Renal Function}

The present study results suggested that renal function influences the plasma level of sLOX-1 in patients with STEMI. Patients with a LOX-1 level above the median were significantly associated with decreased eGFR. Furthermore, there was a significant inverse correlation between plasma sLOX-1 and eGFR. The pathophysiological mechanism by which eGFR was decreased in patients with sLOX-1 above the median remains unclear. The association between lower eGFR and higher plasma sLOX-1 could be in part related to reduced renal clearance of sLOX-1.

\section{Possible Pharmacological Intervention to Lower Plasma sLOX-1 Level}

Previous in vivo and in vitro studies provided evidence that LOX-1 expression can be modulated by various pharmacological treatments and could thus represent an attractive target for therapeutic intervention to limit vascular dysfunction and left ventricular remodeling. Lipid-lowering 3-hydroxy-3-methyl- glutaryl coenzyme A reductase inhibitors (statins) are known to downregulate LOX-1. ${ }^{26,27}$ Pravastatin is reported to downregulate LOX-1 expression in cultured human macrophages and smooth muscle cells. ${ }^{27}$ Upregulation of LOX-1 expression by angiotensin II can be also inhibited in endothelial cells by angiotensin II type 1 receptor blockers. ${ }^{28}$ Clinical trials have demonstrated that these agents are able to reduce the incidence of cardiovascular events after ACS. It would be interesting to test whether these agents will decrease the level of sLOX-1 after STEMI. However, our study showed no differences in medications before admission and at discharge between patients with plasma sLOX-1 levels below and above the median. Further development of specific antagonists is awaited to clarify the precise role of LOX-1, and to investigate the therapeutic potential as an antagonist for post-STEMI patients.

\section{Study Limitations}

This was a single-center study with a relatively small number of patients in the acute phase of STEMI. The prognosis of the patients undergoing primary PCI was relatively favorable, with 22 all-cause deaths and 19 MACE during the follow-up period. Therefore, the ability to predict all-cause death or MACE might be limited. Further large-scale multicenter studies are needed to validate the prognostic value of plasma SLOX-1 after STEMI.

\section{Conclusions}

The incidences of all-cause mortality and MACE in patients with STEMI were significantly and independently associated with the plasma sLOX-1 level on admission. Although further clinical studies are required, measurement of plasma sLOX-1 may be useful to identify patients at high risk for future cardiovascular events.

\section{Disclosures}

None.

\section{Funding}

None.

\section{References}

1. Libby P. Current concepts of the pathogenesis of the acute coronary syndromes. Circulation 2001; 104: 365-372.

2. Falk E, Shah PK, Fuster V. Coronary plaque disruption. Circulation 1995; 92: 657-671.

3. Ross R. Atherosclerosis: An inflammatory disease. N Engl J Med 1999; 340: $115-126$.

4. Steinberg D. Lewis A: Conner Memorial Lecture: Oxidative modification of LDL and atherogenesis. Circulation 1997; 95: 1062-1071.

5. Norata GD, Tonti L, Roma P, Catapano AL. Apoptosis and proliferation of endothelial cells in early atherosclerotic lesions: Possible role of oxidized LDL. Nutr Metab Cardiovasc Dis 2002; 12: 297 305 .

6. Sawamura T, Kume N, Aoyama T, Moriwaki H, Hoshikawa H, Aiba $\mathrm{Y}$, et al. An endothelial receptor for oxidized low-density lipoprotein. Nature 1997; 386: 73-77.

7. Kataoka H, Kume N, Miyamoto S, Minami M, Morimoto M, Hayashida K, et al. Oxidized LDL modulates Bax/Bcl-2 through the lectin like Ox-LDL receptor-1 in vascular smooth muscle cells. Arterioscler Thromb Vasc Biol 2001; 21: 955-960.

8. Kume N, Kita T. Apoptosis of vascular cells by oxidized LDL: Involvement of caspases and LOX-1, and its implication in atherosclerotic plaque rupture. Circ Res 2004; 94: 269-270.

9. Li D, Liu L, Chen H, Sawamura T, Ranganathan S, Mehta JL. LOX-1 mediates oxidized low-density lipoprotein-induced expression of matrix metalloproteinases in human coronary artery endothelial cells. Circulation 2003; 107: 612-617.

10. Kume N, Murase T, Moriwaki H, Aoyama T, Sawamura T, Masaki $\mathrm{T}$, et al. Inducible expression of lectin-like oxidized LDL receptor-1 in vascular endothelial cells. Circ Res 1998; 83: 322-327. 
11. Kataoka H, Kume N, Miyamoto S, Minami M, Moriwaki H, Murase $\mathrm{T}$, et al. Expression of lectin like oxidized low-density lipoprotein receptor-1 in human atherosclerotic lesions. Circulation 1999; 99: 3110-3117.

12. Murase T, Kume N, Kataoka H, Minami M, Sawamura T, Masaki T, et al. Identification of soluble forms of lectin-like oxidized LDL receptor-1. Arterioscler Thromb Vasc Biol 2000; 20: 715-720.

13. Hayashida K, Kume N, Murase T, Minami M, Nakagawa D, Inada $\mathrm{T}$, et al. Serum soluble lectin-like oxidized low-density lipoprotein receptor-1 levels are elevated in acute coronary syndrome: A novel marker for early diagnosis. Circulation 2005; 112: 812-818.

14. Kume N, Mitsuoka H, Hayashida K, Tanaka M, Kita T. Soluble lectin-like oxidized low-density lipoprotein receptor-1 predicts prognosis after acute coronary syndrome: A pilot study. Circ J 2010; 74: 1399-1404.

15. Nakamura M, Ohta H, Kume N, Hayashida K, Tanaka M, Mitsuoka $\mathrm{H}$, et al. Generation of monoclonal antibodies against a soluble form of lectin-like oxidized low-density lipoprotein receptor-1 and development of a sensitive chemiluminescent enzyme immunoassay. $J$ Pharm Biomed Anal 2010; 51: 158-163.

16. Kojima S, Matsui K, Ogawa H, Kumamoto Acute Coronary Events (KACE) Study Group. Temporal trends in hospitalization for acute myocardial infarction between 2004 and 2011 in Kumamoto, Japan. Circ J 2013; 77: 2841-2843.

17. Hao K, Yasuda S, Takii T, Ito Y, Takahashi J, Ito K, et al. Urbanization, life style changes and the incidence/in-hospital mortality of acute myocardial infarction in Japan: Report from the MIYAGI-AMI Registry Study. Circ J 2012; 76: 1136-1144.

18. Nakatani D, Sakata Y, Suna S, Usami M, Matsumoto S, Shimizu M, et al. Incidence, predictors, and subsequent mortality risk of recurrent myocardial infarction in patients following discharge for acute myocardial infarction. Circ J 2013; 77: 439-446.

19. Daida H, Miyauchi K, Ogawa H, Yokoi H, Matsumoto M, Kitakaze $\mathrm{M}$, et al. Management and two-year long-term clinical outcome of acute coronary syndrome in Japan: Prevention of atherothrombotic incidents following ischemic coronary attack (PACIFIC) registry. Circ J 2013; 77: 934-943.

20. Kobayashi N, Takano M, Hata N, Kume N, Yamamoto M, Yokoyama
S, et al. Soluble lectin-like oxidized LDL receptor-1 (sLOX-1) as a valuable diagnostic marker for rupture of thin-cap fibroatheroma: Verification by optical coherence tomography. Int J Cardiol 2013; 168: $3217-3223$.

21. Kobayashi N, Hata N, Kume N, Shinada T, Tomita K, Shirakabe A, et al. Soluble lectin-like oxidized LDL receptor-1 and high-sensitivity troponin $\mathrm{T}$ as diagnostic biomarkers for acute coronary syndrome: Improved values with combination usage in emergency rooms. Circ J 2011; 75: 2862-2871.

22. Takaya T, Wada H, Morimoto T, Sunagawa Y, Kawamura T, Takanabe-Mori R, et al. Left ventricular expression of lectin-like oxidized low-density lipoprotein receptor-1 in failing rat hearts. Circ J 2010; 74: 723-729.

23. Iwai-Kanai E, Hasegawa K, Sawamura T, Fujita M, Yanazuma T, Toyokuni S, et al. Activation of lectin-like oxidized low-density lipoprotein receptor-1 induces apoptosis in cultured neonatal rat cardiac myocytes. Circulation 2001; 104: 2948-2954.

24. Kataoka K, Hasegawa K, Sawamura T, Fujita M, Yanazume T, Toyokuni S, et al. LOX-1 pathway affects the extent of myocardial ischemia-reperfusion injury. Biochem Biophys Res Commun 2003; 300: $656-660$

25. Li L, Sawamura T, Renier G. Glucose enhances endothelial LOX-1 expression: Role for LOX-1 in glucose-induced human monocyte adhesion to endothelium. Diabetes 2003; 52: 1843-1850.

26. Draude G, Hrboticky N, Lorenz RL. The expression of the lectin-like oxidized low-density lipoprotein receptor (LOX-1) on human vascular smooth muscle cells and monocytes and its down-regulation by lovastatin. Biochem Pharmacol 1999; 57: 383-386.

27. Hofnagel O, Luechtenborg B, Eschert H, Weissen-Plenz G, Severs NJ, Robenek H. Pravastatin inhibits expression of lectin-like oxidized low-density lipoprotein receptor-1 (LOX-1) in Watanabe heritable hyperlipidemic rabbits: A new pleiotropic effect of statins. Arterioscler Thromb Vasc Biol 2006; 26: 604-610.

28. Li DY, Zhang YC, Philips MI, Sawamura T, Mehta JL. Upregulation of endothelial receptor for oxidized low-density lipoprotein (LOX-1) in cultured human coronary artery endothelial cells by angiotensin II type 1 receptor activation. Circ Res 1999; 84: 1043-1049. 